\title{
Relación entre satisfacción laboral y engagement en empleados operativos de la empresa de transporte Cotrascal S. A.S.
}

Relationship between job satisfaction and engagement in operative employees of the transport company Cotrascal S. A. S.

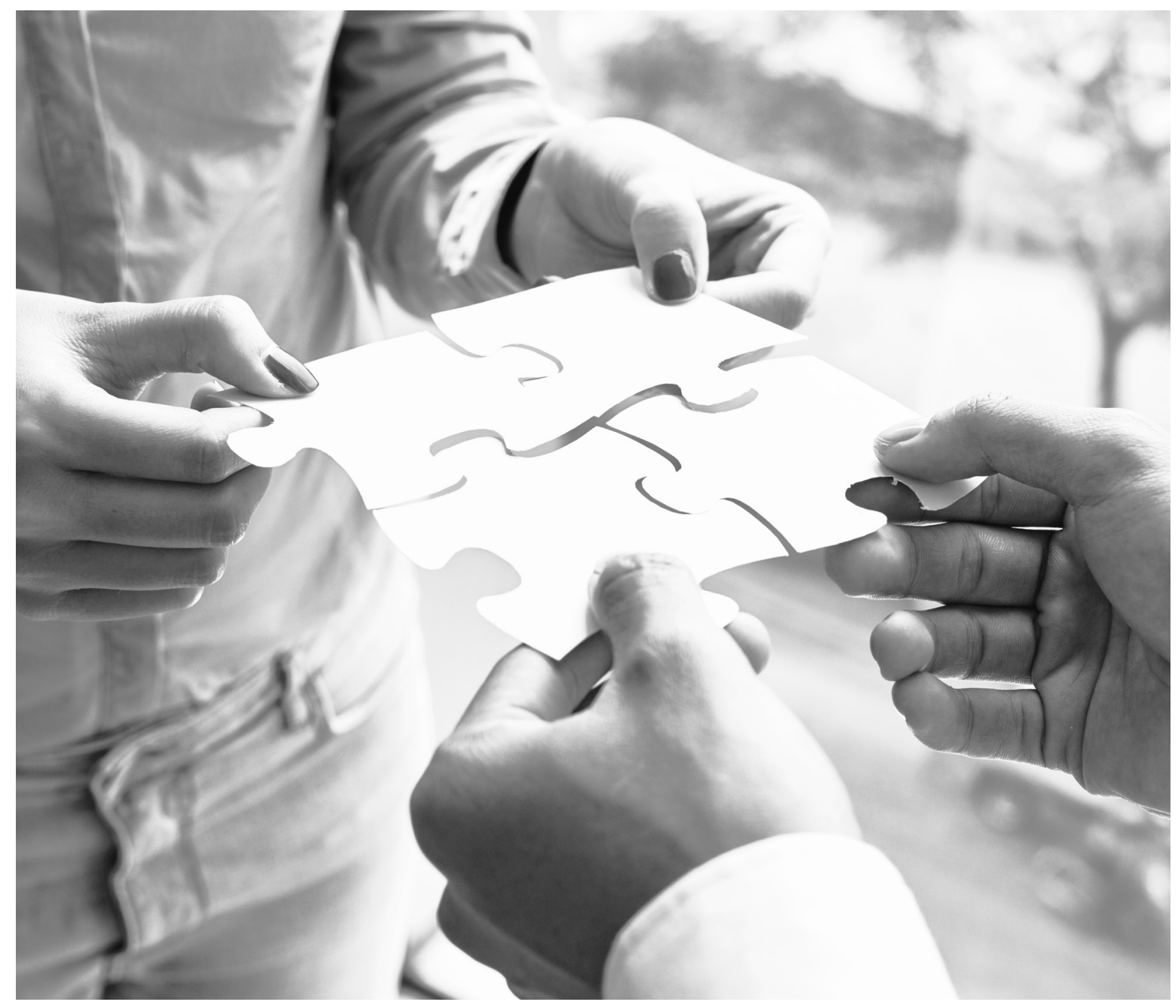




\title{
Relación entre satisfacción laboral y engagement en empleados operativos de la empresa de transporte Cotrascal S. A. S. ${ }^{1}$ Relationship between job satisfaction and engagement in operative employees of the transport company Cotrascal S. A. S.
}

\author{
Robert Fabián Mendoza-Vega ${ }^{2}$, Elsa Gissela Murillo-Murillo³ ${ }^{3}$ Sebastián García-Méndez ${ }^{4}$
}

Artículo recibido en octubre 20 de 2019; artículo aceptado en noviembre 18 de 2019

Este artículo puede compartirse bajo la licencia Licencia Creative Commons Atribución-NoComercial-Compartir lgual 4.0 Internacional y se referencia usando el siguiente formato: Mendoza-Vega, R. F., Murillo-Murillo, E. G \& García-Méndez, S. (2020).

Relación entre satisfacción laboral y engagement en empleados operativos de la empresa de transporte Cotrascal S.A.S.

I+D Revista de Investigaciones, 15 (1), 76-84. DOI: https://doi.org/10.33304/revinv.v15n 1-2020008

\begin{abstract}
Resumen
El presente documento muestra la relación entre satisfacción laboral y engagement en empleados operativos de la empresa de transporte Cotrascal S. A. S., de Bucaramanga. Para el desarrollo de la investigación, se determinó la satisfacción laboral con las variables condiciones laborales, participación en las decisiones, relación con los superiores, trabajo y reconocimiento, mediante la aplicación del cuestionario de satisfacción laboral S20/23. Por su parte, el nivel de engagement se determinó con las dimensiones vigor, dedicación y absorción, aplicando el cuestionario UWES-17. Finalmente se revisó la relación entre estas mediante la correlación de Spearman, teniendo en cuenta la no normalidad de las variables. Los hallazgos indican que se debe fomentar el cierre de brechas entre trabajador y empleador, con el fin de impactar favorablemente los niveles de engagement. Asimismo, se vislumbra una instancia posterior que incluye el componente de percepción del cliente externo mediado por variables internas.
\end{abstract}

Palabras clave: Correlación, engagement, satisfacción laboral, sector transporte.

\footnotetext{
Abstract

This document shows the relationship between job satisfaction and engagement in operative employees of the transport company Cotrascal S. A. S. of Bucaramanga. For the development of the investigation, the job satisfaction

${ }^{1}$ Artículo de investigación, de tipo correlacional, de corte transversal, con enfoque mixto (cuantitativo y cualitativo), resultado de un proyecto de investigación en curso, perteneciente al área de dirección empresarial, subárea de comportamiento organizacional y gestión de recursos humanos, desarrollado en la Universidad de Investigación y Desarrollo (Bucaramanga, Colombia). Dirección: calle 9 n..$^{\circ}$ 23-55, PBX: 6352525. Fecha de inicio: febrero de 2019.

${ }^{2}$ Ingeniero industrial. Universidad de Investigación y Desarrollo (Bucaramanga, Colombia). Dirección: calle 9 n. ${ }^{\circ} 23-55$, PBX: 6352525. ORCID ID: https://orcid.org/0000-0002-2712-9539. Correo electrónico: f_abian_315@hotmail.com.

${ }^{3}$ Ingeniera industrial. Universidad de Investigación y Desarrollo. Jefe de planeación en la Universidad de Investigación y Desarrollo (Bucaramanga, Colombia): Dirección: calle 9 n. ${ }^{\circ}$ 23-55, PBX: 6352525. ORCID ID: https://orcid.org/0000-0003-2441-1093. Correo electrónico: gisselamurillom@ hotmail.com.

${ }^{4}$ Magíster en Administración. Grupo de investigación Sinergia. Profesor de la Universidad de Investigación y Desarrollo (Bucaramanga, Colombia). Dirección: calle 9 n. ${ }^{\circ}$ 23-55, PBX: 6352525. ORCID ID: https://orcid.org/0000-0002-6077-0248. Correo electrónico institucional: sgarcia15@udi.edu.co.
} 
is determined with four variables: working conditions, participation in decision making, relationships with superiors, and work and recognition, through the application of the Job Satisfaction Questionnaire S20/23. Moreover, the level of engagement is determined with the vigor, dedication and absorption dimensions, applying the UWES-17 questionnaire. Finally, the relationship between these was reviewed through the Spearman's correlation, taking into account the non-normality of the variables. The findings indicate that the closing of worker-employer gaps should be encouraged, in order to have a favorable impact on levels and commitment. This raises the prospect of a subsequent instance that includes the external customer perception component, mediated by internal variables.

Keywords: Correlation, engagement, job satisfaction, transport sector.

\section{Introducción}

En el contexto organizacional, el papel y el valor que se le atribuye al recurso humano es, sin lugar a dudas, el principal y más significativo, dado que los colaboradores en una empresa constituyen la carta de presentación para el cliente. Una elevada satisfacción de los empleados en su trabajo conduce al desarrollo con calidad de la prestación de servicios (López \& Labori Ruiz, 2012). Es por esto que, desde la dirección empresarial, se deben enfocar esfuerzos en estrategias que permitan motivar al personal y conocer qué tan comprometido está con las funciones asignadas. Lo anterior, con el propósito de obtener un excelente servicio transmitido al cliente, proporcionándole una experiencia placentera por la atención prestada o por los bienes adquiridos, con lo que se logra posicionar el nombre de la organización y se genera competitividad en el mercado.

Por tanto, en el ámbito del talento humano, la satisfacción laboral juega un papel primordial, pues está directamente relacionada con los niveles de rentabilidad y productividad empresarial (Duarte Payán, Aguirre Ochoa \& Jarquín López, 2014). La satisfacción organizacional tiene por objeto comprender el comportamiento del individuo en el interior de una empresa, influenciado por diferentes factores inherentes a las actividades diarias, lo que posibilita estados emocionales placenteros o positivos en la experiencia del trabajo realizado. Dentro de los factores que influyen se encuentran los altos ingresos, horarios flexibles, oportunidades de progreso, disfrute del trabajo y estabilidad laboral (Duarte Payán et al., 2014). Asimismo, se presenta el engagement, término proveniente del inglés que se asocia, en el contexto empresarial, con el nivel de compromiso, vinculación e implicación laboral que tiene el personal de la empresa para con ella. Sus principales características son el vigor, la dedicación y la absorción (Medrano, Moretti \& Ortiz, 2015).

Por otra parte, Gamboa Gelvez, (2016) en su tesis doctoral titulada "El sector transporte de carga en Santander y sus retos frente a la competitividad del departamento", concluye que existe un retraso a corto plazo con el transporte multimodal para los diversos sectores, que se refleja en las vías que lo conectan. Esto conduce al crecimiento de la informalidad y a la baja competitividad, teniendo en cuenta la continua guerra de precios entre las empresas formales y no formales, lo que mitiga las buenas prácticas y la integridad de los conductores.

En este contexto, en la ciudad de Bucaramanga se encuentra la empresa Cotrascal S. A. S., dedicada al transporte y servicio logístico de mercancías, mediante soluciones sencillas, eficaces y oportunas a los clientes. Esta empresa cuenta además con personal capacitado e idóneo en los diferentes puestos y departamentos. Sin embargo, no se encuentra exenta de las falencias que ha presentado el sector en los últimos años. Se ha enfrentado a la presencia de múltiples quejas en atención al cliente, ocasionadas por inconformidades y demoras en los procesos.

Por esto, en el presente documento se intenta analizar la relación existente entre la satisfacción organizacional y el engagement en el personal operativo de la empresa de transporte Cotrascal S. A. S, con el propósito de formular acciones de mejora. Este proyecto se desarrolla por medio de la aplicación del cuestionario de satisfacción laboral S20/23 y el cuestionario UWES-17, mediante el análisis de correlación de Spearman.

\section{Satisfacción laboral}

Diversos autores coinciden en que la satisfacción laboral es la actitud, sentimiento o perspectiva favorable o desfavorable que tienen los empleados hacia su trabajo, como resultado de la percepción subjetiva de sus experiencias laborales (Blum \& Naylor, 1982; Chiavenato, 1986; Morillo Moronta, 2006; Sikula, 1991; Spector, 1997). Al ser favorable, dicha actitud presenta consecuencias positivas, ya que facilita la satisfacción con la tarea propia; potencia las capacidades; fomenta el rendimiento, la competitividad y la productividad; mejora el clima laboral, y potencializa la autonomía y autorrealización del personal, lo que beneficia la organización (Huilcapi Masacon, Castro López, \& Jácome Lára, 2017).

Para objeto de esta investigación, se empleó la definición de satisfacción laboral de los autores Davis y Newstrom (2003), que la presentan como un conjunto de 
sentimientos y emociones positivas, en los que el agrado por realizar una labor es motivo para sentirse satisfecho y complacido con las actividades desarrolladas en el día a día. En el marco de esta definición se cataloga la satisfacción como los sentimientos y emociones que una persona experimenta a partir de su desarrollo en un entorno determinado.

\section{Tipos de satisfacción laboral}

Herzberg (1976), psicólogo del trabajo, sugiere, con su teoría de los dos factores o teoría del factor dual, que la satisfacción laboral se encuentra conformada por dos tipos de factores: los extrínsecos, del entorno de trabajo, y los intrínsecos, que reflejan las experiencias de los individuos con dicho entorno.

- Satisfacción por factores intrínsecos o motivacionales: Aborda aspectos como la sensación de logro, el reconocimiento interno y el estar a gusto con la tarea desempeñada. En definitiva, deriva de las recompensas de índole interno al propio individuo ofrecidas por su trabajo. Los factores motivacionales provocan satisfacción, pero su ausencia no genera insatisfacción.

- Satisfacción por factores extrínsecos o higiénicos: Proviene de factores tales como las políticas de la organización, la supervisión, las condiciones laborales y salariales, la relación con los compañeros y la conciliación de la vida profesional y personal. Estos factores son motivo de insatisfacción, por lo que resulta necesario cuidarlos.

\section{Indicadores de la satisfacción laboral}

A partir de las diferentes investigaciones realizadas sobre satisfacción laboral, múltiples autores, como Bòria-Reverter, Crespi-Vallbona y Mascarilla-Miró (2012), Calvalcante Silva (2004) y Pichler y Wallace (2009) han logrado identificar un conjunto de variables que se encuadran en el ámbito de los determinantes de la satisfacción y surgen a partir de los tipos de satisfacción ya mencionados. Dichas variables se describen a continuación:

- Relaciones con los superiores: Incluyen el estilo de liderazgo o las capacidades técnicas y administrativas de los jefes o superiores, así como cualidades a nivel de relacionamiento interpersonal de estos. Es importante que los directivos actúen como líderes motivadores, puesto que esto ayudará a mejorar la relación en los departamentos o áreas de trabajo para el buen funcionamiento de las labores y cumplimiento oportuno de las metas propuestas.

- Condiciones laborales: Se refieren a los sistemas de recompensas, el sueldo y la promoción. Estos son factores con gran influencia e importancia para la satisfacción del trabajador, ya que permiten cubrir los gastos correspondientes a sus necesidades fisiológicas y estilo de vida. Además, devengar un buen sueldo es símbolo de reconocimiento y seguridad. Por otra parte, también hace referencia al ambiente físico laboral: limpieza, condiciones ambientales, ergonómicas y saludables.

- Participación en las decisiones: Resulta ser un aspecto clave para el desarrollo de la iniciativa e independencia del individuo. Cuanto mayor es el control percibido por este sobre la situación del trabajo, mayor será su satisfacción, ya que la posibilidad de ser partícipe en las decisiones de un grupo fomenta la motivación, promueve la integración y conduce al consenso grupal.

- Naturaleza del trabajo: Se refiere al tipo de ocupación, el puesto de trabajo o la jerarquía que ocupan los trabajadores. Se ha demostrado en el ámbito global que desarrollar un trabajo atractivo e interesante es uno de los factores más valorados en relación con la satisfacción, y asimismo, que el departamento en el que se trabaja también puede resultar un factor a considerar.

- Reconocimiento laboral: Comprende los elogios o críticas al trabajo realizado, así como las recompensas percibidas debido a la evaluación justa del desempeño laboral. Aspectos como este incentivan y motivan al personal; y cuanto más se potencian, mejores resultados se obtienen de los empleados.

\section{Método de medición de la satisfacción laboral}

Existen diferentes métodos de evaluación para determinar la satisfacción laboral que presentan los trabajadores de una organización. Entre estos, se destaca por su uso el cuestionario $54 / 82$, de 82 ítems, que muestra satisfactorios valores de fiabilidad y validez. Es un cuestionario extenso, robusto en contenido, útil como fuente de diagnóstico, preciso en investigación y en consultoría (Meliá, Peiró \& Calatallud, 1986). Sin embargo, para la presente investigación se decidió emplear el cuestionario S20/23, que consta de 23 ítems y puede considerarse como la versión reducida más completa de las desarrolladas a partir del S4/82. La versión S20/23 presenta unas instrucciones sensiblemente más breves y sencillas que la versión $\$ 4 / 82$ y mantiene las mismas alternativas de respuesta (de 1: Muy insatisfecho a 7: Muy satisfecho) (Meliá \& Peiró, 1989).

El cuestionario $\mathrm{S} 20 / 23$ presenta un nivel favorable de fiabilidad y validez, a saber, un alfa de Cronbach de 0,92 (Meliá, Pradilla, Martí, Sancerni, Oliver \& Tomás, 1990), lo que permite la obtención de una medida global de 
satisfacción y la descripción de los cinco indicadores de satisfacción laboral descritos en el apartado anterior. El S20/23 ha sido diseñado teniendo en cuenta las importantes restricciones motivacionales y temporales a las que puede estar expuesto el trabajo del investigador en contextos organizacionales (Meliá \& Peiró, 1989).

\section{El engagement}

El engagement laboral es definido por múltiples autores como un estado mental positivo y satisfactorio frente al trabajo, determinado por el vigor, la dedicación y la absorción del trabajador en el desarrollo de las actividades laborales (Bakker, Demerouti \& Xanthopoulou, 2011; Salanova \& Schaufeli, 2009; Schaufeli, Salanova, GonzálezRomá \& Bakker, 2002; Wefald, 2008). Dicho de otra forma, el engagement es el sentimiento de responsabilidad, compromiso y motivación de los trabajadores frente al desempeño de su trabajo (Bakker et al., 2011; Britt, 1999).

Así mismo, otras investigaciones han demostrado que los empleados engaged son personas optimistas y eficaces por sí mismas, capaces de manejar las situaciones que les presenta el día a día. Debido a la actitud positiva y alto nivel de diligencia, los empleados son capaces de generar retroalimentación propia, en cuanto a valoración, reconocimiento y éxito (Forbes, 2013). Los empleados experimentan continuamente emociones, como la alegría y el entusiasmo, que posiblemente amplían el proceso de pensamiento y acción de las personas. Por esta razón, están en constante aprendizaje y adquieren nuevas habilidades que los llevan a trabajar interiormente en los propios recursos personales, los que a su vez se emplean en el desarrollo de las actividades laborales (Bakker et al., 2011).

\section{Dimensiones del engagement}

Las dimensiones del engagement son un planteamiento trifactorial basado en la psicología positivista que permite entender el concepto más ampliamente y facilitar su estudio. Según los principales autores que han abordado este tema de investigación (Bakker et al., 2011; Salanova \& Schaufeli, 2009; Schaufeli et al., 2002), estas dimensiones se pueden definir como:

- Vigor: Se caracteriza por los altos niveles de energía y resistencia mental mientras se trabaja, por el deseo de esforzarse en la tarea que se está realizando, incluso cuando se presentan dificultades.

- Dedicación: Hace referencia a una alta implicación laboral, junto con la manifestación de un sentimiento de entusiasmo, inspiración, orgullo y reto por el trabajo.

- Absorción: Corresponde a la percepción sobre el premio o incentivo recibido por el trabajo bien hecho.

El objetivo final de alcanzar las tres dimensiones dentro de la empresa es lograr obtener cambios positivos para la organización y para los empleados. A su vez, mejorar las expectativas y percepciones que estos tengan de sus puestos de trabajo y también lograr una alta satisfacción, consolidando experiencias gratificantes que les permitan un óptimo desarrollo, tanto personal como profesional.

\section{Instrumentos de medición de engagement}

El planteamiento trifactorial de las dimensiones del engagement, basado en la psicología positivista, ha sido medido a través de diversos instrumentos, entre los cuales se destaca la Escala Utrecht de Engagement en el Trabajo -o UWES, por sus siglas en inglés-. La propuesta original del UWES contaba con 24 ítems; sin embargo, se eliminaron 7 reactivos defectuosos, y quedó finalmente constituido por 17 ítems (UWES-17), cuyo alfa de Cronbach en las dimensiones oscila entre 0,82 y 0,91 (Schaufeli, Bakker, Valdéz \& Ron, 2003). Lo anterior indica que los factores vigor, dedicación y absorción se constituyen como indicadores de engagement (Schaufeli et al., 2002). Asimismo, sobresale el Inventario de Burnout de Oldenburg -u OLBI, por sus siglas en inglés- como herramienta de medición de engagement laboral, aunque este inicialmente fue creado para evaluar el burnout, que se define como un síndrome con connotaciones afectivas negativas que afecta a los trabajadores a niveles sociales, personales y laborales (Salanova, 2006). Por lo anterior, algunas corrientes han entendido el engagement como el estado opuesto del burnout. Por esto, considerando que esta escala tiene preguntas planteadas tanto negativa como positivamente, puede ser también utilizada para medir el engagement. Se mide desde el vigor hasta el agotamiento, y de la dedicación hasta el cinismo, pero apartando la absorción como descriptor de cualquiera de estas conductas (Organización Mundial de la Salud, 2013).

\section{Correlación de Spearman}

Como resultado de la revisión literaria, se puede definir el coeficiente de correlación de Spearman como una medida de asociación entre variables de índole cuantitativo, que no tiene en cuenta el nivel de acuerdo o concordancia entre estas (no paramétrica). Este tipo de correlación es recomendable cuando los datos presentan valores extremos, ya que dichos valores afectan el coeficiente de correlación de Pearson, o ante distribuciones no normales (Santander Montes \& Ruiz Vaquero, 2004).

\section{Cotrascal S. A. S.}

La empresa Cotrascal S. A. S. fue fundada en el año 2001. Actualmente cuenta con 8 sedes en el ámbito nacional, (Bucaramanga, Bogotá, Medellín, Cúcuta, Valledupar, 
Barranquilla, Dos Quebradas y Yumbo). Esta empresa está dedicada a brindar soluciones y servicios en el sector transporte de carga terrestre y logística. Cuenta adicionalmente con un talento humano comprometido con la satisfacción de los clientes, el beneficio mutuo y el progreso de la empresa. Además, se reconoce con gran potencial de crecimiento para los próximos años.

\section{Metodología}

\section{Tipo de estudio}

El presente estudio se consolidó bajo el enfoque mixto (cualimétrico), con el fin de recolectar, analizar y vincular datos cuantitativos y cualitativos (Gómez, 2015) para responder al planteamiento entre los elementos a estudiar de satisfacción laboral y engagement. La investigación es de tipo correlacional, de corte transversal e implica la sistematización de las relaciones entre las variables de una muestra específica para ser analizada de forma estadística.

\section{Procedimiento}

Para el presente estudio se realiza la medición de la satisfacción laboral de los trabajadores operativos de la empresa Cotrascal S. A. S., a partir del cuestionario S20/23. Posteriormente se evalúa el engagement mediante el instrumento UWES-17. Finalmente, para determinar la relación existente entre satisfacción laboral y el engagement se analizan los coeficientes de correlación de Spearman entre las variables analizadas de cada uno.

\section{Participantes}

En el estudio participaron 100 empleados operativos de la empresa Cotrascal S. A. S. El $68 \%$ de los trabajadores que hicieron parte de esta investigación son hombres. En cuanto al estado civil de los encuestados, el $24 \%$ es casado; el $44 \%$, soltero, y el $32 \%$ se encuentra en unión libre. Además, el principal nivel de escolaridad es bachillerato $(47 \%)$, mientras que en menor medida los empleados son tecnólogos (12\%), técnicos (27\%) y profesionales (14\%).

\section{Materiales e instrumentos}

Los instrumentos de recolección de información sobre la satisfacción laboral y el engagement seleccionados para este estudio fueron el cuestionario S20/23, de Meliá y Peiró (1989), y el UWES-17, de Schaufeli et al. (2002) respectivamente, que han sido ampliamente utilizados en diferentes investigaciones en los ámbitos nacional e internacional, y cuentan con respuestas tipo Likert de 7 niveles. En estas se tuvieron en cuenta las siguientes categorías: 1 corresponde a Muy insatisfecho; 2, a Bastante insatisfecho; 3, a Algo insatisfecho; 4, a Indiferente; 5, a Algo satisfecho; 6, a Bastante satisfecho, y 7, a Muy satisfecho.

\section{Resultados}

Los resultados presentados a continuación relacionan la satisfacción laboral, el engagement y finalmente los coeficientes de correlación de Spearman para las variables objeto de estudio en la empresa Cotrascal S. A. S.

\section{Satisfacción laboral}

La satisfacción laboral de la empresa Cotrascal S. A. S. en gran nivel se encuentra satisfecha, debido a que las condiciones de los trabajadores y los procesos que se desarrollan en las actividades del día a día cumplen con los requisitos del personal que labora en la organización (ver Tabla 1). Al analizar cada dimensión, se observa que la variabilidad sobre esta es mínima, puesto que la empresa objeto de estudio cumple con los requerimientos del empleado con un nivel de formación tecnóloga, porque son los que se encuentran en su totalidad muy satisfechos con el trabajo, las condiciones laborales y el reconocimiento otorgado por la empresa (ver Tabla 2). Dichos patrones fueron determinados con base en el instrumento de recolección de la información aplicado.

Tabla 1

Satisfacción laboral

\begin{tabular}{ccc}
\hline Dimensiones & Puntaje & $\begin{array}{c}\text { Nivel de } \\
\text { satisfacción }\end{array}$ \\
\hline $\begin{array}{c}\text { Condiciones laborales } \\
\text { Participación en las } \\
\text { decisiones }\end{array}$ & 5,83 & $\begin{array}{c}\text { Bastante } \\
\text { satisfecho }\end{array}$ \\
Relación con los superiores & 6,25 & $\begin{array}{c}\text { Muy satisfecho } \\
\text { Muy satisfecho } \\
\text { Trabajo }\end{array}$ \\
Reconocimiento & 5,98 & $\begin{array}{c}\text { Bastante } \\
\text { satisfecho } \\
\text { Bastante } \\
\text { satisfecho }\end{array}$ \\
\hline
\end{tabular}

Fuente: Autores.

Tabla 2

Satisfacción laboral por unidad de análisis

\begin{tabular}{|c|c|c|}
\hline Unidad de análisis & Puntaje & $\begin{array}{c}\text { Nivel de } \\
\text { satisfacción }\end{array}$ \\
\hline Género masculino & 5,85 & $\begin{array}{l}\text { Bastante } \\
\text { satisfecho }\end{array}$ \\
\hline Género femenino & 5,69 & $\begin{array}{l}\text { Bastante } \\
\text { satisfecho }\end{array}$ \\
\hline Estado civil casado(a) & 6,01 & Muy satisfecho \\
\hline Estado civil soltero(a) & 5,85 & $\begin{array}{l}\text { Bastante } \\
\text { satisfecho }\end{array}$ \\
\hline Estado civil unión libre & 5,61 & $\begin{array}{l}\text { Bastante } \\
\text { satisfecho }\end{array}$ \\
\hline Profesional & 5,69 & $\begin{array}{l}\text { Bastante } \\
\text { satisfecho }\end{array}$ \\
\hline Tecnólogo & 6,06 & Muy satisfecho \\
\hline Técnico & 5,74 & $\begin{array}{l}\text { Bastante } \\
\text { satisfecho }\end{array}$ \\
\hline Bachiller & 5,83 & $\begin{array}{c}\text { Bastante } \\
\text { satisfecho }\end{array}$ \\
\hline
\end{tabular}

Fuente: Autores. 


\section{Engagement}

Así mismo, el nivel de engagement en los empleados operativos de la empresa Cotrascal S. A. S. es bastante alto, pues, según los resultados de este estudio, se encuentran muy comprometidos con el trabajo realizado en el lugar que desarrollan sus funciones y enfocados en cumplir con el objetivo trazado por la organización (ver Tabla 3).

Tabla 3

Niveles de engagement

\begin{tabular}{ccc}
\hline Dominios & Puntaje & $\begin{array}{c}\text { Nivel de } \\
\text { Engagement }\end{array}$ \\
\hline Vigor & 6,47 & $\begin{array}{c}\text { Muy } \\
\text { comprometido } \\
\text { Muy } \\
\text { Dedicación }\end{array}$ \\
Absorción & 6,53 & $\begin{array}{c}\text { comprometido } \\
\text { Muy } \\
\text { comprometido }\end{array}$ \\
\hline
\end{tabular}

Fuente: Autores.

En cuanto a los resultados que muestra la Tabla 4, se puede observar cómo el nivel de engagement es medianamente proporcional al nivel o formación académica de los trabajadores, pues el puntaje del profesional es ligeramente mayor al del técnico o bachiller, lo que reafirma lo encontrado en la teoría concerniente a la naturaleza del trabajo, en la que se resalta que, según el cargo o jerarquía, los trabajadores pueden desarrollar mayor satisfacción laboral (Bòria-Reverter et al., 2012). Por consiguiente, se infiere que depende en su mayoría directamente del nivel de formación académica.

Tabla 4

Niveles de engagement por unidad de análisis

\begin{tabular}{|c|c|c|}
\hline Unidad de análisis & Puntaje & $\begin{array}{c}\text { Nivel de } \\
\text { Engagement }\end{array}$ \\
\hline Género masculino & 6,34 & $\begin{array}{c}\text { Muy } \\
\text { comprometido }\end{array}$ \\
\hline Género femenino & 6,34 & $\begin{array}{c}\text { Muy } \\
\text { comprometido }\end{array}$ \\
\hline Estado civil casado(a) & 6,40 & $\begin{array}{c}\text { Muy } \\
\text { comprometido }\end{array}$ \\
\hline Estado civil soltero(a) & 6,34 & $\begin{array}{c}\text { Muy } \\
\text { comprometido }\end{array}$ \\
\hline Estado civil: unión libre & 6,34 & $\begin{array}{c}\text { Muy } \\
\text { comprometido }\end{array}$ \\
\hline Profesional & 6,42 & $\begin{array}{c}\text { Muy } \\
\text { comprometido }\end{array}$ \\
\hline Tecnólogo & 6,41 & $\begin{array}{c}\text { Muy } \\
\text { comprometido }\end{array}$ \\
\hline Técnico & 6,33 & $\begin{array}{c}\text { Muy } \\
\text { comprometido }\end{array}$ \\
\hline Bachiller & 6,35 & $\begin{array}{c}\text { Muy } \\
\text { comprometido }\end{array}$ \\
\hline
\end{tabular}

Fuente: Autores.

\section{Correlación de Spearman}

Con el objetivo de establecer la relación existente entre las dimensiones de las variables engagement y satisfacción laboral, se hallan los coeficientes de correlación de Spearman, que se presentan en la Tabla 5. Estos coeficientes de correlación se encuentran entre el rango 0,071 y 0,331. Al llevarse a cabo un análisis de los resultados obtenidos, se evidencia que existe un alto grado de relación entre las dimensiones vigor y relación con los superiores $(0,331)$, y vigor y trabajo $(0,331)$. Dichas relaciones se presentan con la misma dimensión de la variable engagement (vigor), por lo que se infiere que las buenas relaciones con los superiores influyen de gran manera en la voluntad de dedicar esfuerzo y empeño a sus labores en el trabajo, ya que estos, desde su rol de líderes, a partir de las buenas relaciones, transmiten ánimo y fuerza para que sus subalternos se sientan a gusto con sus funciones. Algo similar ocurre con los objetivos y metas que se tracen desde sus cargos, pues estos son de gran relevancia para fijar el grado de satisfacción con el que se desempeña un trabajador.

Tabla 5

Correlación de Spearman entre las variables de satisfacción laboral y engagement

\begin{tabular}{cccc}
\hline & Vigor & Dedicación & Absorción \\
\hline $\begin{array}{c}\text { Condiciones } \\
\text { laborales }\end{array}$ &, $201^{*}$ & 0,071 & 0,141 \\
$\begin{array}{c}\text { Participación en } \\
\text { las decisiones }\end{array}$ &, $299^{* *}$ &, $328^{* *}$ &, $292^{* *}$ \\
$\begin{array}{c}\text { Relación con los } \\
\text { superiores }\end{array}$ &, $331^{* *}$ &, $301^{* *}$ &, $328^{* *}$ \\
$\quad$ Trabajo &, $331^{* *}$ &, $297^{* *}$ & 0,174 \\
Reconocimiento &, $317^{* *}$ & 0,195 &, $299^{* *}$ \\
\hline
\end{tabular}

Fuente: Autores.

\section{Conclusiones}

La satisfacción de los empleados de la empresa Cotrascal S. A. S. se encuentra en niveles bastante alto y muy satisfecho. Particularmente es necesario recalcar la dimensión de relación con los superiores, que permite inferir que las líneas jerárquicas no son rígidas en el plano organizacional, lo que posibilita un entorno idóneo para el desarrollo de las diversas funciones de los empleados. Por otra parte, se debe examinar al detalle el componente de reconocimiento en el marco de fidelizar el recurso humano.

Por su parte, el engagement de los empleados de la empresa Cotrascal S. A. S. presenta niveles altos. Por tal motivo, se debe visualizar que el recurso humano tiene gran alineación con la empresa, lo que posibilita el logro de los objetivos organizacionales en determinados periodos temporales. 
La relación entre las variables objeto de estudio permite visualizar que la dimensión de engagement más relacionada es la concerniente al vigor. Por su parte, la dimensión de satisfacción laboral con más influencia es la referente a los superiores. Por consiguiente, se infiere la importancia de la cohesión del equipo de trabajo tanto operativo como administrativo en el marco de la gestión del personal y potencial percepción exitosa del cliente interno. Asimismo, se recalca la importancia de eliminar barreras del organigrama, con el fin de generar mejores condiciones de trabajo.

\section{Recomendaciones}

En aras de mejorar de forma continua y generar altos índices de competitividad, la organización debe propiciar condiciones de trabajo favorables para sus trabajadores, ya que esto influye significativamente en el compromiso con el que ejercen sus labores, $y$, además, en el grado de satisfacción con el que llevan a cabo su trabajo.

La satisfacción laboral y el engagement son ejes fundamentales del trabajo en el marco de la gestión de los procesos del talento humano en el interior de las organizaciones. Por tal motivo, se recomienda que la empresa Cotrascal S. A. S. implemente prácticas con relación a las diversas unidades de estudio, con el fin de generar escenarios idóneos de operación que apalanquen indicadores del proceso y contribuyan a la potencial satisfacción del cliente externo.

Así mismo, a partir de los hallazgos encontrados, se sugiere fomentar el cierre de brechas entre trabajador y empleador, con el fin de impactar favorablemente los niveles de engagement.

También sería interesante replicar este análisis en empresas de otros sectores, utilizando el componente de percepción del cliente externo mediado por variables internas, o realizarlo mediante la implementación de otros métodos de medición para las variables de satisfacción laboral o engagement.

\section{Referencias}

Bakker, A. B., Demerouti, E., \& Xanthopoulou, D. (2011). Cómo los empleados mantienen su engagement en el trabajo? Ciencia \& Trabajo, 13(4), 135-142.

Blum, M., \& Naylor, J. (1982). Psicología Industrial (Sus Fundamentos Teóricos y Sociales) (Trillas, Ed.). México, D.F.

Bòria-Reverter, S., Crespi-Vallbona, M., \& Mascarilla-Miró, O. (2012). Determining variables of job satisfaction in Spain. Cuadernos de Economia, 35(97), 9-16. https:// doi.org/10.1016/S0210-0266(12)70018-3

Britt, T. W. (1999). Engaging the self in the field: Testing the triangle model of responsibility. Personality and
Social Psychology Bulletin, 25(6), 698-708.

Calvalcante Silva, J. J. (2004). Satisfacción en el trabajo de los directores de escuelas secundarias públicas. Universidad Autonoma de Barcelona.

Chiavenato, I. (1986). Administración de Humanos (McGraw Hill, Ed.). México, D.F.

Davis, K., \& Newstrom, J. W. (2003). Comportamiento humano en el trabajo (McGraw Hill, Ed.).

Duarte Payán, S. M., Aguirre Ochoa, J., \& Jarquín López, G. E. (2014). Factores que influyen en la satisfacción laboral de los empleados de la empresa Adara S.A. Revista Electrónica de Investigación En Ciencias Económicas, 2(4), 20-33.

Forbes, R. (2013). La psicología organizacional positiva y la mejora organizacional. Exito Empresarial, 1(227), 1-3. Recuperado de http://www.cegesti.org /exitoempresarial/ publicaciones/publicacion_227_040313_es.pdf

Gamboa Gelvez, D. C. (2016). El sector transporte de carga en Santander y sus retos frente a la competitividad del departamento. Universidad Industrial de Santander.

Gómez, M. C. S. (2015). La dicotomía cualitativocuantitativo: posibilidades de integración y diseños mixtos. Campo Abierto. Revista de Educación, 1(1), 11-30.

Herzberg, F. (1976). The managerial choice: To be efficient and to be human. Irwin Professional Publishing.

Huilcapi Masacon, M., Castro López, G., \& Jácome Lára, G. (2017). Motivación: las teorías y su relación en el ámbito empresarial. Dominio de Las Ciencias, 3(2), 311-333.

López, M. R., \& Labori Ruiz, J. R. (2012). Satisfacción por los servicios brindados en la sala de Fisioterapia del Hospital General "Dr. Juan Bruno Zayas Alfonso" de Santiago de Cuba. Medisan, 16(08), 1235-1240.

Medrano, L. A., Moretti, L., \& Ortiz, A. (2015). Medición del engagement académico en estudiantes universitarios. Revista Iberoamericana de Diagnóstico y Evaluación-e Avaliação Psicológica, 2(40), 114-124.

Meliá, J. L., \& Peiró, J. M. (1989). La medida de la satisfacción laboral en contextos organizacionales: El Cuestionario de Satisfacción S20/23. Psicologemas, 5, 59-74.

Meliá, J. L., Peiró, J. M., \& Calatallud, C. (1986). El Cuestionario General de Satisfacción Laboral en contextos organizacionales laborales. Estudios factoriales, fiabilidad y validez (presentación del cuestionario S4/82). Millars, 9, 43-77.

Meliá, J. L., Pradilla, J. F., Martí, N., Sancerni, M. D., Oliver, A., \& Tomás, J. M. (1990). Estructura factorial, fiabilidad y validez del cuestionario de satisfacción S21/26. Un instrumento con formato dicotómico orientado al trabajo profesional. Universitas Tarraconensis, 12, 39.

Morillo Moronta, I. (2006). Nivel de satisfacción del personal académico del Instituto Pedagógico de Miranda José Manuel Siso Martínez en relación con el estilo de liderazgo del jefe del departamento, las relaciones interpersonales de los miembros adscritos y el sistema de incentivo. Sapiens: Revista 
Universitaria de Investigación, 7(1), 43-58.

Organización Mundial de la Salud. (2013). Ambientes de trabajo saludables: un modelo para la acción.

Pichler, F., \& Wallace, C. (2009). What are the Reasons for differences in job satisfaction across Europe? Individual, compositional, and institutional explanations. European Sociological Review, 25(5), 535-549.

Salanova, M. (2006). Medida y evaluación del burnout: nuevas perspectivas. "El síndrome de quemarse por el trabajo en Servicios Sociales".

Salanova, M., \& Schaufeli, W. (2009). El engagement en el trabajo: cuando el trabajo se convierte en pasión (Alianza Editorial, Ed.). Madrid.

Santander Montes, A., \& Ruiz Vaquero, R. (2004). Relación entre variables cuantitativas (Cuba: ECIMED, Ed.).

Schaufeli, W., Bakker, A., Valdéz, H., \& Ron, C. (2003). Escala Utrecht de Engagement en el trabajo. Valencia: Utrecht Univesity.

Schaufeli, W. B., Salanova, M., González-Romá, V., \& Bakker, A. B. (2002). The measurement of engagement and burnout: A two sample confirmatory factor analytic approach. Journal of Happiness Studies, 3(1), 71-92.

Sikula, A. F. (1991). Administración de recursos humanos en empresas (Limusa, Ed.). México, D.F.

Spector, P. E. (1997). Job satisfaction: Application, assessment, causes, and consequences (Sage publications, Ed.). London.

Wefald, A. J. (2008). An examination of job engagement, transformational leadership, and related psychological constructs. Kansas State University. 